\title{
Modeling the heterogeneity in risk of progression to Alzheimer's disease across cognitive profiles in mild cognitive impairment
}

\author{
Curtis Tatsuoka ${ }^{1,2,3^{*}}$, Huiyun Tseng ${ }^{4}$, Judith Jaeger ${ }^{5,6}$, Ferenc Varadi ${ }^{7}$, Mark A Smith ${ }^{8}$, Tomoko Yamada ${ }^{1}$,
} Kathleen A Smyth ${ }^{1,3}$, Alan J Lerner ${ }^{1,2}$ and The Alzheimer's Disease Neuroimaging Initiative

\begin{abstract}
Introduction: Heterogeneity in risk of conversion to Alzheimer's disease (AD) among individuals with mild cognitive impairment $(\mathrm{MCl})$ is well known. Novel statistical methods that are based on partially ordered set (poset) models can be used to create models that provide detailed and accurate information about performance with specific cognitive functions. This approach allows for the study of direct links between specific cognitive functions and risk of conversion to $\mathrm{AD}$ from $\mathrm{MCl}$. It also allows for further delineation of multi-domain amnestic $\mathrm{MCl}$, in relation to specific non-amnestic cognitive deficits, and the modeling of a range of episodic memory functioning levels.
\end{abstract}

Methods: From the Alzheimer's Disease Neuroimaging Initiative (ADNI) study, conversion at 24 months of $268 \mathrm{MCl}$ subjects was analyzed. It was found that 101 of those subjects (37.7\%) converted to AD within that time frame. Poset models were then used to classify cognitive performance for $\mathrm{MCl}$ subjects. Respective observed conversion rates to $A D$ were calculated for various cognitive subgroups, and by APOE e4 allele status. These rates were then compared across subgroups.

Results: The observed conversion rate for $\mathrm{MCl}$ subjects with a relatively lower functioning with a high level of episodic memory at baseline was $61.2 \%$. In MCl subjects who additionally also had relatively lower perceptual motor speed functioning and at least one APOE e4 allele, the conversion rate was $84.2 \%$. In contrast, the observed conversion rate was $9.8 \%$ for $\mathrm{MCl}$ subjects with a relatively higher episodic memory functioning level and no APOE e4 allele. Relatively lower functioning with cognitive flexibility and perceptual motor speed by itself also appears to be associated with higher conversion rates.

Conclusions: Among MCl subjects, specific baseline cognitive profiles that were derived through poset modeling methods, are clearly associated with differential rates of conversion to AD. More precise delineation of $\mathrm{MCl}$ by such cognitive functioning profiles, including notions such as multidomain amnestic $\mathrm{MCl}$, can help in gaining further insight into how heterogeneity arises in outcomes. Poset-based modeling methods may be useful for providing more precise classification of cognitive subgroups among $\mathrm{MCl}$ for imaging and genetics studies, and for developing more efficient and focused cognitive test batteries.

\section{Introduction}

There is increasing interest in the recognition and treatment of prodromal stages of Alzheimer's disease (AD), especially mild cognitive impairment (MCI). MCI is viewed as a state between normal cognitive functioning

\footnotetext{
* Correspondence: cmt66@case.edu

'Department of Neurology, Case Western Reserve University, 11100 Euclid

Avenue, Cleveland, $\mathrm{OH} 44106$ USA

Full list of author information is available at the end of the article
}

and dementia. Those with MCI are characterized as exhibiting mild problems with memory and/or other cognitive functions, while still being able to perform daily life activities normally or nearly so [1]. Despite having a higher overall risk of developing $\mathrm{AD}$ [1], conversion outcomes among those with $\mathrm{MCI}$ are quite heterogeneous. Only about $15 \%$ of these individuals convert 
to $\mathrm{AD}$ per year; many never convert, and some revert to normal cognition [2]. This heterogeneity makes it difficult to design efficient trials of agents designed to delay or prevent progression from MCI to $\mathrm{AD}$ or to interpret the outcomes of these trials [3], or to evaluate potential AD biomarkers. Hence, there is a clear need to better delineate cognitive phenotypes in MCI.

Although MCI subgroups that reflect deficit heterogeneity, such as amnestic single domain MCI, amnestic multidomain $\mathrm{MCI}$, and non-amnestic multidomain $\mathrm{MCI}$ [4] have been developed, they lack specificity in the particular cognitive functions that are impaired in each subgroup. This type of specification is challenging because neuropsychological (NP) response data are complex. It can be difficult to isolate a deficit in a particular cognitive function, since performing well on most NP measures requires tapping into several cognitive functions, and it is often not possible to design tests that tap one cognitive domain to the exclusion of all others. For example, it is possible to perform poorly on a verbal list-learning task as a result of impaired attention or word fluency, and in the absence of an amnestic disturbance. Hence, if an individual performs poorly on a given measure, it may be difficult to pinpoint exactly which function is impaired.

Subscales are commonly used in an attempt to improve specificity in analysis of NP assessment data. For instance, subscales can be derived from factor analysis through the use of factor scores. However, scale-based approaches are generally limited by the assumption of a direct correspondence between a subscale score and an associated function. Poor performance on a subscale is interpreted as indicating a deficit in the function the subscale is purported to measure, even if the poor performance is due to deficits in functions not associated with the subscale. This makes it difficult to link NP test performance to specific functional deficits, and hence to identify cognitive phenotypes that can be linked to outcomes such as conversion from MCI to AD.

Use of total scores on multi-item measures, such as the Alzheimer's disease assessment scale-cognitive (ADASCog) measure [5] or the mini-mental status exam (MMSE) [6], as a basis for cognitive phenotyping is also problematic. Total scores represent a (weighted) sum of response scores from items assessing several cognitive domains. However, the same total score can be derived from a range of different response patterns, without regard to the cognitive functions being assessed by each item. In this sense, items are viewed as interchangeable, even though the cognitive targets of assessment for the items can vary considerably. Because a wide range of response patterns and cognitive interpretations can give rise to the same score, resulting phenotypes lack specificity.

\section{The partially ordered set (poset) modeling approach to interpreting NP data}

Poset models serve as a basis for novel methods tailored for classifying the performance (that is, functioning) levels of subjects with respect to specific cognitive functions. Classification is conducted based on observed responses to NP measures. Each measure is associated with specific cognitive functions that are involved in performing well on that measure, so that the ties between observed responses and functioning levels are clear. This approach is feasible because posets have essential statistical convergence properties such as assuring that a subject's state is identified accurately with sufficient measurement, even in the presence of measures that are associated with multiple functions. Theoretically derived validation tools are available as well. Statistical theory and data-analytic frameworks for the poset approach have been established in Tatsuoka and Ferguson (2003) [7] and Tatsuoka (2002) [8].

In this paper, our goal is to demonstrate that posets can improve our understanding of MCI heterogeneity. The modeling results in the development of states associated with profiles of cognitive functioning that summarize performance levels for each of the cognitive functions being tested by a given NP battery. Hence, a state can be viewed as similar to a diagnostic classification in which the diagnosis represents a particular pattern of cognitive strengths and weaknesses. States are ordered by comparing the associated performance levels for each of the functions included in the analysis. One state is considered greater than a second state if its associated performance level on at least one function is strictly higher than the performance level for the second state, and its performance levels for all other functions are at least as high. However, posets are flexible in that it is not necessary that one state be greater than another, in other words, the states can be partially ordered. This arises when one state in comparison to another state has a higher performance level with respect to one function, while having a lower performance level with respect to another function. This enables models to reflect a complex range of responses from an NP battery.

A probability distribution on the states is used to represent belief about which state best describes the cognitive capabilities of a subject. Bayes' rule is used to obtain updated posterior probabilities of state membership once responses to measures are observed. This allows for a systematic manner in which the information obtained from observing multiple measures can be combined for statistical classification. Two response distributions are estimated per NP measure, one representing the response tendencies of subjects who perform at a relatively high level on all functions associated with the particular measure and another for those subjects who 
do not. These distributions are used to weigh the relative likelihood of an observed response indicating that a subject has the associated higher level functioning. The approach of estimating two response distributions per NP measure is parsimonious in that there is no need to estimate response distribution parameters that are specific to each profile; rather, response distribution parameters are shared either by profiles that have the associated functioning levels, or those that do not. Ideally, one state in the model will have a probability close to 1 for each individual, while other states will have probabilities near 0 , indicating that a subject's cognitive profile is known with near certainty. As long as models are correctly specified, this near certainty will indeed be obtained given a sufficient amount of testing [7]. Once classification is completed, subjects who share a cognitive profile can be aggregated, and observed rates of conversion to $\mathrm{AD}$ between the resulting subgroups can be compared.

In our study, we used NP data collected by the Alzheimer's Disease Neuroimaging Initiative (ADNI) [9] to evaluate the usefulness of poset models to identify specific cognitive phenotypes associated with conversion from MCI to $\mathrm{AD}[7,8,10,11]$. We hypothesized that poset modeling would generate interpretable and sufficiently detailed cognitive phenotypes with clearly differentiated rates of conversion from MCI to AD. Given its established importance in progression risk for $\mathrm{AD}$, Apolipoprotein E (APOE) e4 status was also taken into account $[12,13]$.

\section{Materials and methods} Study sample

MCI subjects enrolled in the ADNI $(n=389)$ were included in the classification analysis if their scores on the selected NP battery were available at both baseline and at 24 months. The sample was $64.5 \%$ male and $93.1 \%$ Caucasian. About 3.8\% were African American, 2.8\% were Asian, and $0.3 \%$ were American Indian or Alaskan Native. Mean age was 74.8 years $(\mathrm{SD}=7.5)$ and mean length of education was 15.7 years $(\mathrm{SD}=3.0)$.

\section{Modeling and classification approach}

We used neuropsychologist expert opinion (JJ and HYT) to map the relationship between selected ADNI NP measures (ADAS delayed recall and word recognition subscales and number cancellation; auditory verbal learning test (AVLT) Trial 6 and List B; Boston naming test; category fluency; trail making Test $\mathrm{A}$ and Test $\mathrm{B}$, and Wechsler adult intelligence scale-revised (WAIS-R) digit symbol substitution) and the cognitive functions required to perform them (episodic memory at four different levels, word fluency, cognitive flexibility, perceptual motor speed, and attention). See Table 1 for the listing of these specifications. Measures were selected based on the types of functions they tested and retained based on statistical criteria such as discriminatory properties and correspondence with model fit. Given the reliance on expert opinion, data-analytic validation is important. Statistical details on how this validation was performed are provided in Additional file 1 (Appendix).

To acknowledge the key role of episodic memory impairment as an early symptom in $\mathrm{AD}$ and to better represent the varying levels of episodic memory required across the measures, we identified four levels of episodic memory proficiency (levels 0 through 3 ). These levels are ordered in terms of episodic memory demand, with level the highest, and level 0 the lowest. Level 3

Table 1 Cognitive functions and how they are tapped into by selected ADNI neuropsychology measures, as specified according to expert opinion

\begin{tabular}{|c|c|c|c|c|c|c|c|}
\hline \multirow[t]{2}{*}{ ADNI measure } & \multicolumn{7}{|c|}{ Cognitive function } \\
\hline & $\begin{array}{c}\text { Episodic memory } \\
\text { level } 1\end{array}$ & $\begin{array}{c}\text { Episodic memory } \\
\text { level } 2\end{array}$ & $\begin{array}{c}\text { Episodic memory } \\
\text { level } 3\end{array}$ & $\begin{array}{c}\text { Word } \\
\text { fluency }\end{array}$ & $\begin{array}{l}\text { Selective } \\
\text { attention }\end{array}$ & $\begin{array}{l}\text { Cognitive } \\
\text { flexibility }\end{array}$ & $\begin{array}{c}\text { Perceptualmotor } \\
\text { speed }\end{array}$ \\
\hline $\begin{array}{l}\text { ADAS-delayed recall } \\
\text { subscale }\end{array}$ & $x$ & $x$ & $x$ & & $x$ & & \\
\hline $\begin{array}{l}\text { ADAS word } \\
\text { recognition subscale }\end{array}$ & $x$ & & & & $x$ & & \\
\hline AVLT-Trial 6 & $x$ & $x$ & $x$ & & $x$ & & \\
\hline AVLT-List B & $x$ & $x$ & & & $x$ & & \\
\hline Boston naming test & & & & $x$ & $x$ & & \\
\hline Category fluency $^{*}$ & & & & $x$ & $x$ & $x$ & \\
\hline $\begin{array}{l}\text { ADAS-number } \\
\text { cancellation }\end{array}$ & & & & & $x$ & & \\
\hline Trail making test $\mathrm{A}$ & & & & & $x$ & & $x$ \\
\hline Trail making test $B$ & & & & & $x$ & $x$ & $x$ \\
\hline $\begin{array}{l}\text { WAIS-R digit symbol } \\
\text { substitution }\end{array}$ & & & & & $x$ & & $x$ \\
\hline
\end{tabular}

ADAS, Alzheimer's Disease Cooperative Study; ADNI, Alzheimer's Disease Neuroimaging Initiative; AVLT, adult verbal learning test; WAIS-R, Wechsler adult intelligence scale-revised; ${ }^{*}$ average of vegetable and animal categories. 
Table 2 Description of episodic memory functioning levels used in the model

\begin{tabular}{ll}
\hline Level & \multicolumn{1}{c}{ Description } \\
\hline Level 3 & Relatively longer-term delayed recall with distractors \\
Level 2 & Relatively shorter recall durations, such as involved in AVLT List B \\
Level 1 & Immediate recall, word recognition \\
Level 0 & Cannot perform well at any of the above levels
\end{tabular}

AVLT, adult verbal learning test

corresponds to relatively longer-term delayed recall with distractors, while level 2 and level 1, respectively, relate to shorter and shorter recall durations involved in AVLT List B and immediate recall. A subject at level 0 cannot perform well at any of the other levels. A brief summary of the practical interpretation of the episodic memory levels to be used here is given in Table 2 .

Through the assumed hierarchical ordering between levels of episodic memory, note that the delayed recall measures that involve level 3 also can provide information on functioning with levels 1 and 2. High functioning at level 3 implies high functioning at levels 1 and 2 as well. Also, poor performance on word recognition, which is associated with level 1 , not only provides evidence that level 1 is at a low level, but that functioning at levels 2 and 3 should be low as well. Hence, even though associations of measures involving episodic memory can be at different levels, they still can inform other levels of episodic memory functioning, affording some degree of replication.

\section{Poset model generation}

A poset model was generated based on the functions associated with each of the ADNI NP measures. In the cognitive profiles associated to states in the model, performance levels for a function were denoted either as relatively high, or relatively low, in relation to the $\mathrm{MCI}$ and early $\mathrm{AD}$ sample. See Table 3 for a complete list of profiles that can

Table 3 Poset states and their corresponding profiles of cognitive functioning relative to mild cognititve impairment and Alzheimer's disease

\begin{tabular}{|c|c|c|c|c|c|c|c|}
\hline \multirow[t]{2}{*}{ Poset state } & \multicolumn{7}{|c|}{ Cognitive function } \\
\hline & $\begin{array}{l}\text { Selective } \\
\text { attention }\end{array}$ & $\begin{array}{c}\text { Episodic memory } \\
\text { level } 1\end{array}$ & $\begin{array}{c}\text { Episodic memory } \\
\text { level } 2\end{array}$ & $\begin{array}{c}\text { Episodic memory } \\
\text { level } 3\end{array}$ & $\begin{array}{c}\text { Word } \\
\text { fluency }\end{array}$ & $\begin{array}{l}\text { Cognitive } \\
\text { flexibility }\end{array}$ & $\begin{array}{c}\text { Perceptual motor } \\
\text { speed }\end{array}$ \\
\hline 1 & $x$ & $x$ & $x$ & $x$ & $x$ & $x$ & $x$ \\
\hline 2 & $x$ & $x$ & $x$ & $x$ & $x$ & $x$ & \\
\hline 3 & $x$ & $x$ & $x$ & $x$ & $x$ & & $x$ \\
\hline 4 & $x$ & $x$ & $x$ & $x$ & $x$ & & \\
\hline 5 & $x$ & $x$ & $x$ & $x$ & & $x$ & $x$ \\
\hline 6 & $x$ & $x$ & $x$ & $x$ & & & $x$ \\
\hline 7 & $x$ & $x$ & $x$ & $x$ & & * & $x$ \\
\hline 8 & $x$ & $x$ & $x$ & & $x$ & $x$ & $x$ \\
\hline 9 & $x$ & $x$ & $x$ & & $x$ & $x$ & \\
\hline 10 & $x$ & $x$ & $x$ & & $x$ & & $x$ \\
\hline 11 & $x$ & $x$ & $x$ & & $x$ & & \\
\hline 12 & $x$ & $x$ & $x$ & & & $x$ & $x$ \\
\hline 13 & $x$ & $x$ & & & $x$ & & $x$ \\
\hline 14 & $x$ & $x$ & $x$ & & & * & \\
\hline 15 & $x$ & $x$ & & & $x$ & $x$ & $x$ \\
\hline 16 & $x$ & $x$ & & & $x$ & $x$ & \\
\hline 17 & $x$ & $x$ & & & $x$ & & $x$ \\
\hline 18 & $X$ & $x$ & & & $x$ & & \\
\hline 19 & $x$ & $x$ & & & & $x$ & $x$ \\
\hline 20 & $x$ & $x$ & & & & & $x$ \\
\hline 21 & $x$ & $x$ & & & & * & \\
\hline 22 & $x$ & & & & $x$ & $x$ & $x$ \\
\hline 23 & $x$ & & & & $x$ & $x$ & \\
\hline 24 & $x$ & & & & $x$ & & $x$ \\
\hline 25 & $x$ & & & & $x$ & & \\
\hline 26 & $x$ & & & & & $x$ & $x$ \\
\hline 27 & $x$ & & & & & & $x$ \\
\hline 28 & $x$ & & & & & * & \\
\hline 29 & & & & & & & \\
\hline
\end{tabular}

$\mathrm{X}$ indicates relatively high functioning; ${ }^{*}$ undetermined.. 
be distinguished by the given set of NP measures that were analyzed.

The resultant poset model comprised 29 states. These states (and associated cognitive profiles) represent the profiles that can be distinguished from the NP battery, and were identified algorithmically from the expertderived specifications in Table 1 [14]. Table 3 shows how the cognitive functions we examined are distributed across states to create distinct cognitive profiles. The corresponding partial ordering of these profiles is graphically depicted in Figure 1 as a Hasse diagram. Note that state 1 is the highest state in the poset, as it has the highest level of functioning for all five cognitive functions. State 29 is the lowest state, since all its functioning for all five functions is at the lowest level. For the in-between states, there exists at least one function for which performance is not at a relatively high level. In the diagram, connected lines between states indicate

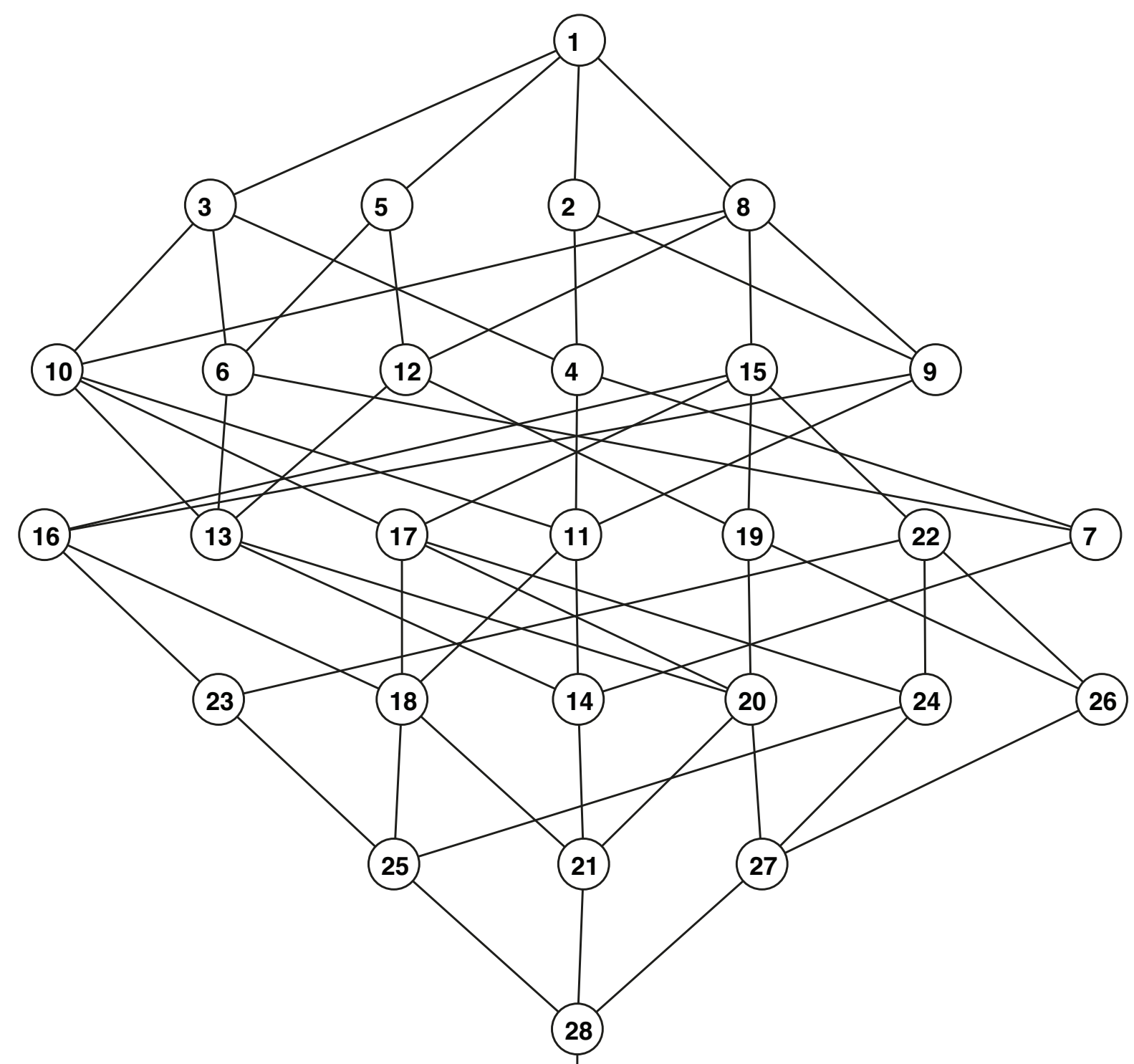

28

Figure 1 Partially ordered set (poset) model for cognitive functioning of mild cognifitive impairment and early Alzheimer's disease subjects. 
direct ordering, with the higher of the states being greater than the lower one.

In our analysis, a uniform prior probability value of $1 / 29$ was assigned to each state for each subject to indicate prior belief about which profile would fit a given subject. We then estimated two response distributions for each NP measure as described above. These distributions were then used to weigh the relative likelihood that an observed response indicated that a subject had the associated higher level functioning.

\section{Response distributions}

Responses from both the MCI subjects and also early AD subjects (an additional 174 such subjects) were included in the estimation, to allow for a range of values. Given the apparent non-normality of response data, nonparametric approaches to response distribution estimation were adopted [10,11,15-17] (see Additional file 1).

\section{Grouping of profiles and classified subjects}

The ordered relationships between states arise when identifying subgroups with shared functioning levels for a function. For instance, the subgroup of states that have high performance level for episodic memory level 2 are all the states greater than or equal to state 14. Precisely, this would be state 1 through 12, and state 14 . The complement of this subgroup (all states not greater than or equal to state 14) would thus comprise the states with lower performance level. Once subgroups such as this have been identified and classification conducted, the probability that a subject has a particular performance level for a function can be computed by summing the posterior probabilities of membership of each of the states in the subgroup. These probabilities are used as a basis for cutoff values in function-related groupings, which are then compared statistically in terms of proportion of AD conversions from MCI. All reported $P$-values are two-sided.

We treated cognitive flexibility slightly differently from other NP functions, due to confounding of its functioning status in classification under certain profiles, specifically for states 7, 14, 21, and 28. Confounded profiles arise due to limitations of the NP battery to distinguish all possible profiles. Profiles with confounding give conflicting information about certain functions, but probabilities for a subject being at certain functioning levels can still be obtained by weighting the information provided across a set of confounded profiles (see Additional file 1 for more details).

\section{Model validation}

Briefly, model fit appears to be good. Response distribution estimates for all measures correspond to the assumed order structure, in that those subjects expected by the model to score well actually tended to do so, and those not expected to score well tended not to do so. Moreover, classification was fairly decisive, especially given the limited number of NP measures employed. Observed responses to the measures were thus consistent with the model specifications. See Figures S1 through S6 and Table S1 in Additional file 1.

\section{Results and discussion}

We explored different cognitive groupings within MCI subjects, to assess conversion rates to $\mathrm{AD}$ at two years post baseline. Not surprisingly, episodic memory is the cognitive function that appears to be most significantly related to future conversion. Still, the different performance levels of episodic memory in our model appear to have varying degrees and ways of association with conversion outcomes. For instance, as seen in Table 4, level 2 episodic memory performance levels influence the rate of conversion among those with MCI. In subjects for whom level 2 episodic memory functioning is low (in other words, below the cutoff probability value of less than 0.275$), 41$ out of $67(61.2 \% \pm 11.7 \%, 95 \% \mathrm{CI})$ converted to $\mathrm{AD}$ within two years, which is much higher than the overall MCI to AD conversion rate in this sample of 101 out of $268(37.7 \% \pm 5.8 \%, 95 \%$ CI). Those with relatively lower performance in level 2 of episodic memory significantly differ in conversion rates compared with those with higher performance, regardless of whether or not the APOE e4 allele is present. The $P$-value for Fisher's exact test is 0.000 for a two-sided test of no association between conversion and having relatively low episodic memory level 2 functioning.

Other functions where relatively lower functioning at baseline may indicate higher risk for conversion from $\mathrm{MCI}$ to $\mathrm{AD}$ are perceptual motor speed and cognitive flexibility. For perceptual motor speed, using a cutoff

Table 4 Relationship between episodic memory level 2 functioning and conversion to Alzheimer's disease (AD) over a two-year period

\begin{tabular}{cccc}
\hline Converted to $\mathbf{A D}$ & Low episodic memory level $\mathbf{2}$ function & Not low episodic memory level 2 function & Total \\
\hline Yes & $61.2 \%(41)$ & $29.9 \%(60)$ & $37.7 \%(101)$ \\
No & $38.8 \%(26)$ & $70.1 \%(141)$ & $62.3 \%(167)$ \\
Total & $100 \%(67)$ & $100 \%(201)$ & $100 \%(268)$ \\
\hline
\end{tabular}

Results are presented as the percent (number) of patients. Fisher's exact test two-sided $P$-value $=0.000$ for a test of no association; positive predictive value $=0.612$; negative predictive value $=0.701$. 
probability value of 0.40 to delineate a lower functioning subgroup, and considering subjects with at least one APOE4 allele, 23 of $35(65.7 \% \pm 15.7 \%$, 95\% CI) of MCI subjects with relatively low functioning convert to $\mathrm{AD}$ within 24 months. On the other hand, only 14 of 35 MCI subjects with relatively low perceptual motor speed and without an APOE4 allele convert $(40 \% \pm 16.2 \%$, 95\% $\mathrm{CI}$ ). Further, for cognitive flexibility, using a cutoff probability value of 0.30 to delineate a lower functioning subgroup, 28 of $48(58.3 \% \pm 13.9 \%, 95 \% \mathrm{CI})$ of MCI subjects with relative low baseline functioning convert to AD within 24 months. In this case, the $P$-value for Fisher's exact test of no association is 0.007 .

Interestingly, we conversely found much lower rates of conversion among certain cognitive profiles. In particular, only four out of forty-one $(9.8 \% \pm 9.1 \%, 95 \% \mathrm{CI}) \mathrm{MCI}$ subjects with no APOE e4 alleles and relatively high level-3 episodic memory functioning (cutoff probability value greater than 0.80 ) convert in two years. This rate appears to be lower than for subjects with no APOE e4 allele but without high level-3 episodic memory functioning ( $P$-value $=0.001$, Fisher's exact test of no association $)$.

\section{Amnestic multidomain $\mathrm{MCl}$ identified with poset models}

An advantage of the poset approach is the ability to provide classification to profiles that address a range of functions. Subgroups, such as amnestic multidomain MCI, can be characterized more precisely by identifying specific functions that are relatively impaired along with episodic memory.

In our sample, subjects with relatively low functioning on episodic memory level 2 (probability of being at high level at level 2 being 0.275 or less) and perception speed (probability cutoff value of 0.40 or less) were identified. As shown in Table 5, among subjects with at least one APOE e4 allele and with relatively low levels of both episodic memory and perception speed, 16 out of 19 converted $(84.2 \% \pm 16.4 \%, 95 \% \mathrm{CI})$. Considering only subjects with lower level-2 episodic memory functioning, those who additionally have lower functioning in perceptual motor speed appear even more likely to convert $(P$-value $=0.013$, Fisher's exact test of no association $)$.
In contrast, additionally having lower functioning with cognitive flexibility did not appear to significantly increase risk for conversion. Among subjects with lower episodic memory level 2 functioning, observed percentages of conversion with and without lower cognitive flexibility were $66.7 \%$ versus $57.5 \%$ (18 out of 27 versus 23 out of 40).

\section{Cognitive change before conversion}

Over the range of cognitive functions, histograms were generated for the respective probabilities of converters having relatively high functioning over the 24 months preceding conversion. Analogous histograms were generated for non-converters over a time period of the same duration starting from baseline. See Figures 2, 3, 4, $5,6,7,8$. Note that for non-converters, only those subjects who did not convert over the full 36-month period of the study were included in the plots. By comparing corresponding plots between converters and non-converters, it becomes clearer how the above findings of heterogeneity in conversion outcomes by cognitive profile arose.

For Figures 2, 3, 4, which correspond to the episodic memory levels, note that relative to level 2, level 1 does not see the same amount of decline over 24 months for converters, as reflected by a shift to lower probability values. Hence, the discrepancy between the histograms in Figures $2 \mathrm{a}$ versus $2 \mathrm{~b}$ over time between converters and non-converters is not very strong. On the other hand, in Figures 3a and 3b, it is clear that for converters, there is quite a bit of decline in level 2 values during this time period, while non-converters appear stable. This makes level 2 attractive for discrimination and prediction over this duration. In Figures $4 \mathrm{a}$ and $4 \mathrm{~b}$, note that level 3 functioning is low among almost all converters preceding conversion. However, lower functioning for this more difficult level also is common for non-converters, lessening the discriminatory properties of level 3. Still, there is a sizeable proportion of non-converters retaining high probability values for level 3 , which allows for cognitively-based identification of a very lowrisk group.

Table 5 Relationship between episodic memory level 2 and perceptual motor speed functioning and conversion to AD over a two-year period by APOE4 allele status

\begin{tabular}{|c|c|c|c|c|c|c|}
\hline \multirow[t]{2}{*}{$\begin{array}{c}\text { Converted to } \\
\text { AD }\end{array}$} & \multicolumn{3}{|c|}{$\begin{array}{l}\text { Low episodic memory function and no } \\
\text { APOE4 } \text { allele }^{\mathrm{a}}\end{array}$} & \multicolumn{3}{|c|}{$\begin{array}{l}\text { Low episodic memory function and at least one } \\
\text { APOE4 allele }{ }^{b}\end{array}$} \\
\hline & $\begin{array}{l}\text { Low perceptual motor } \\
\text { speed function }\end{array}$ & $\begin{array}{l}\text { Not low perceptual } \\
\text { motor speed function }\end{array}$ & Total & $\begin{array}{l}\text { Low perceptual motor } \\
\text { speed function }\end{array}$ & $\begin{array}{l}\text { Not low perceptual } \\
\text { motor speed Function }\end{array}$ & Total \\
\hline Yes & $63.6 \%(7)$ & $54.5 \%(6)$ & $59.1 \%(13)$ & $84.2 \%(16)$ & $37.8 \%$ 17) & $61.2 \%(18)$ \\
\hline No & $36.4 \%$ (4) & $45.5 \%(5)$ & 40.9\% (9) & $15.8 \%$ (3) & $62.2 \%(28)$ & $38.8 \%(10)$ \\
\hline Total & $100 \%(11)$ & $100 \%(11)$ & $100 \%(22)$ & $100 \%$ (19) & $100 \%(13)$ & $100 \%(28)$ \\
\hline
\end{tabular}

Results are presented as the percent (number) of patients. ${ }^{\mathrm{a} F i s h e r}$ 's exact test two-sided $P$-value $=1.00$ for a test of no association. ${ }^{\mathrm{b}}$ Fisher's exact test two-sided $P$-value $=0.013$ for a test of no association. AD, Alzheimer's disease; APOE, Apolipoprotein. 
(a)

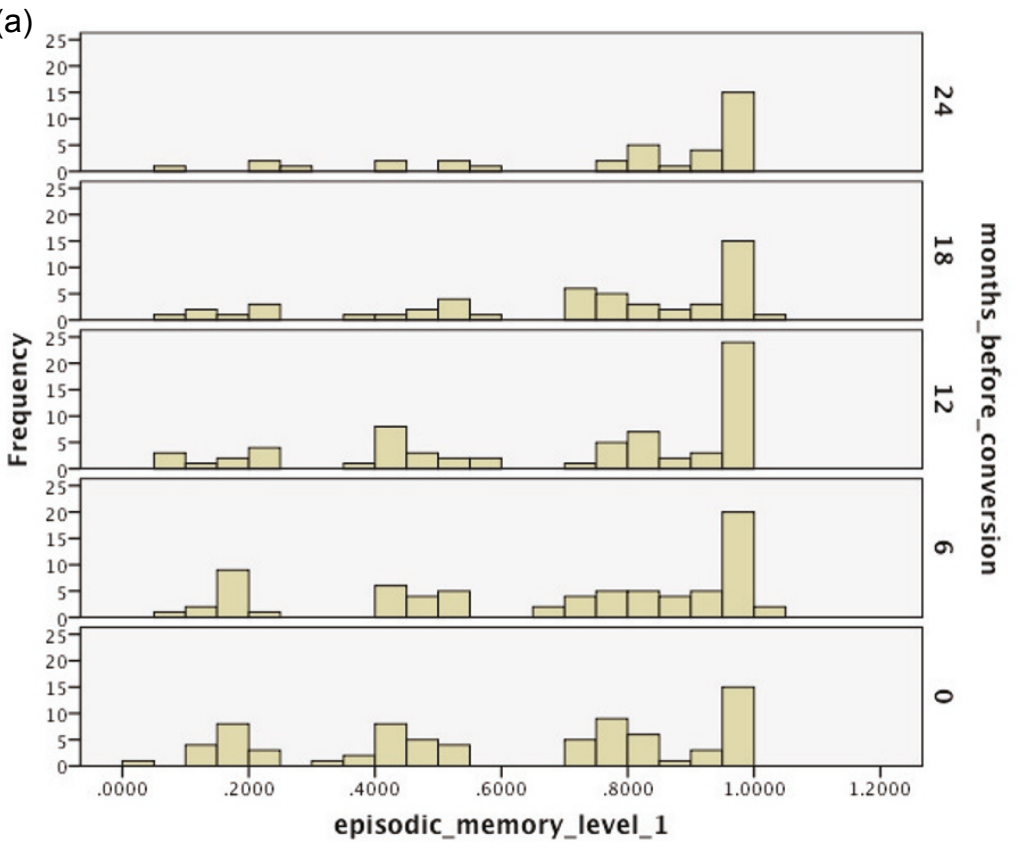

(b)

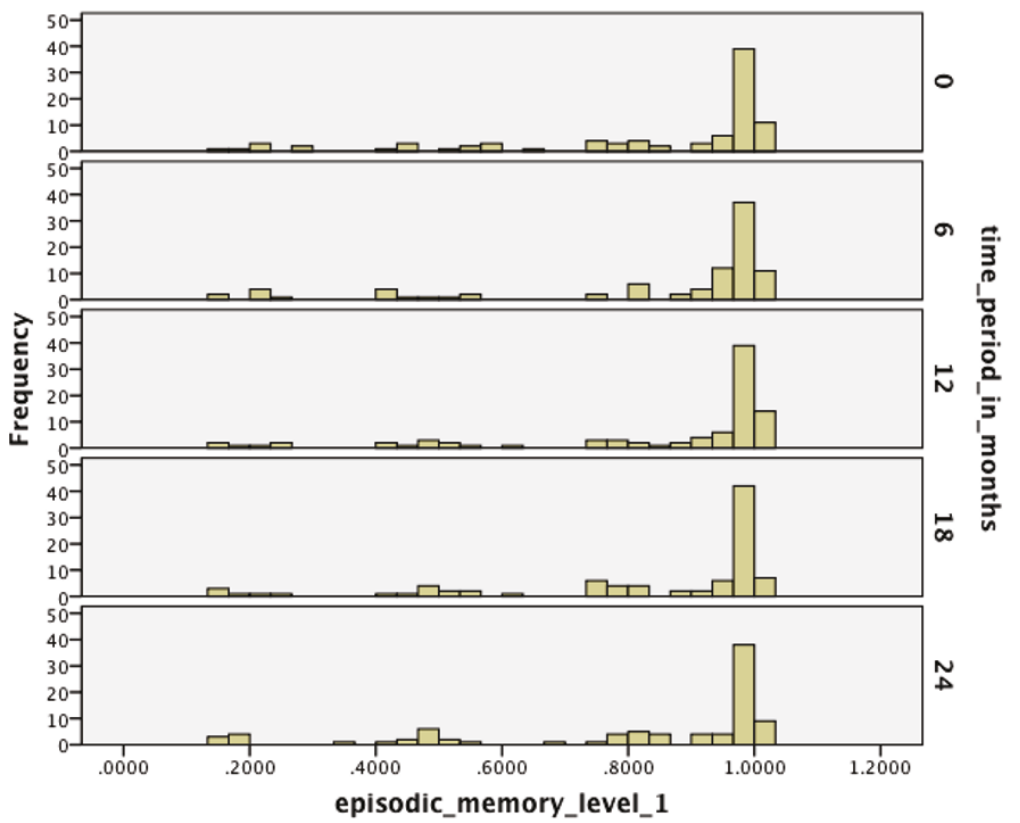

Figures 2 Probabilities of functioning over time among mild cognitive impairment (MCI) converters to Alzheimer's disease (AD) versus MCI non-converters. (a) Probability of relatively high functioning with episodic memory level 1 from 24 to 0 months before conversion to AD among MCl subjects. Respectively, $n=75,75,68,51$, and 36 for $0,6,12,18$ and 24 months before conversion. (b) Probability of relatively high functioning with episodic memory level 1 from baseline (0 months) to 24 months among MCl subjects who did not convert within 36 months; $n=90$, 


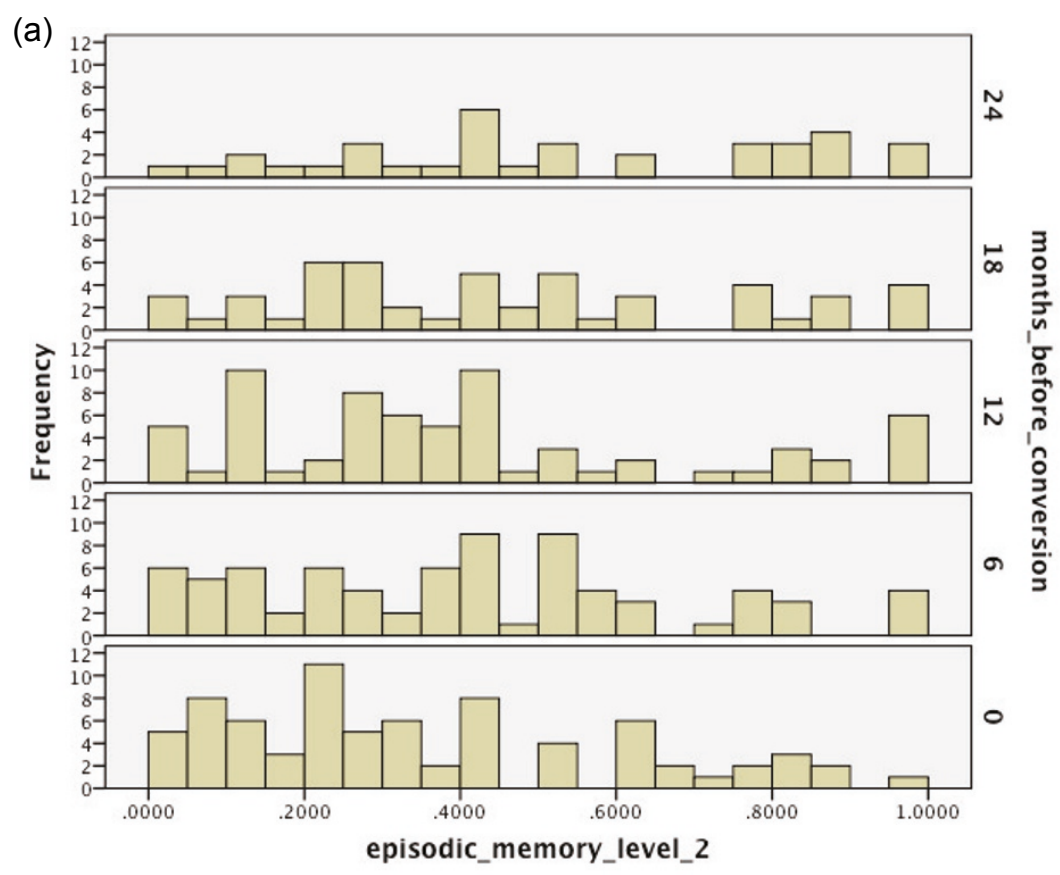

(b)

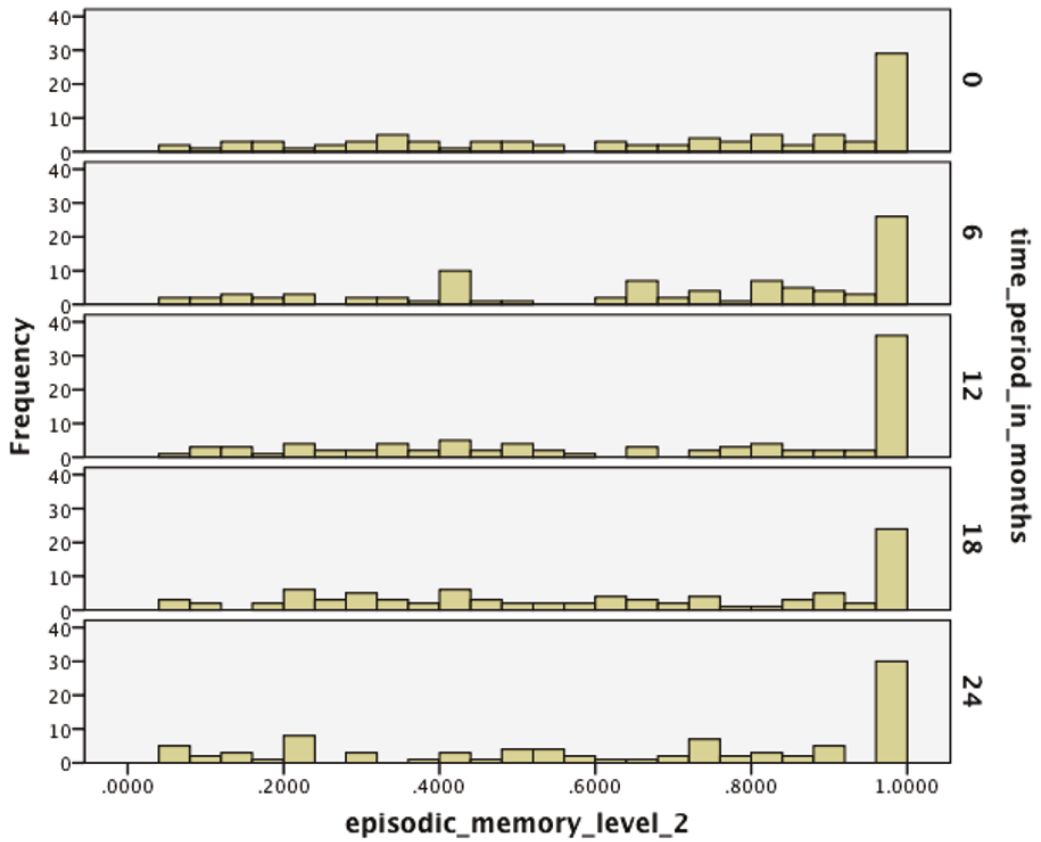

Figure 3 Probabilities of functioning over time among mild cognitive impairment (MCl) converters to Alzheimer's disease (AD) versus MCI non-converters. (a) Probability of relatively high functioning with episodic memory level 2 from 0 to 24 months before conversion to AD among $\mathrm{MCl}$ subjects. (b) Probability of relatively high functioning with episodic memory level 2 from baseline to 24 months among MCl subjects who did not convert within 36 months. 


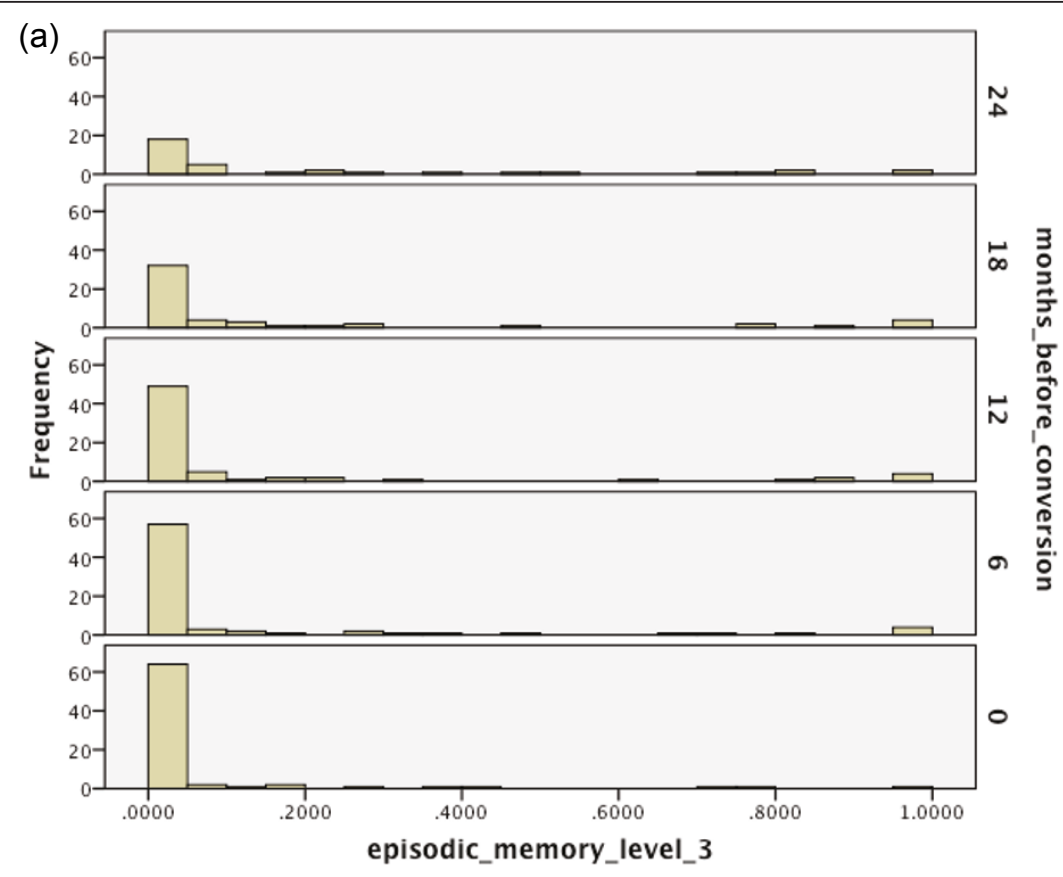

(b)

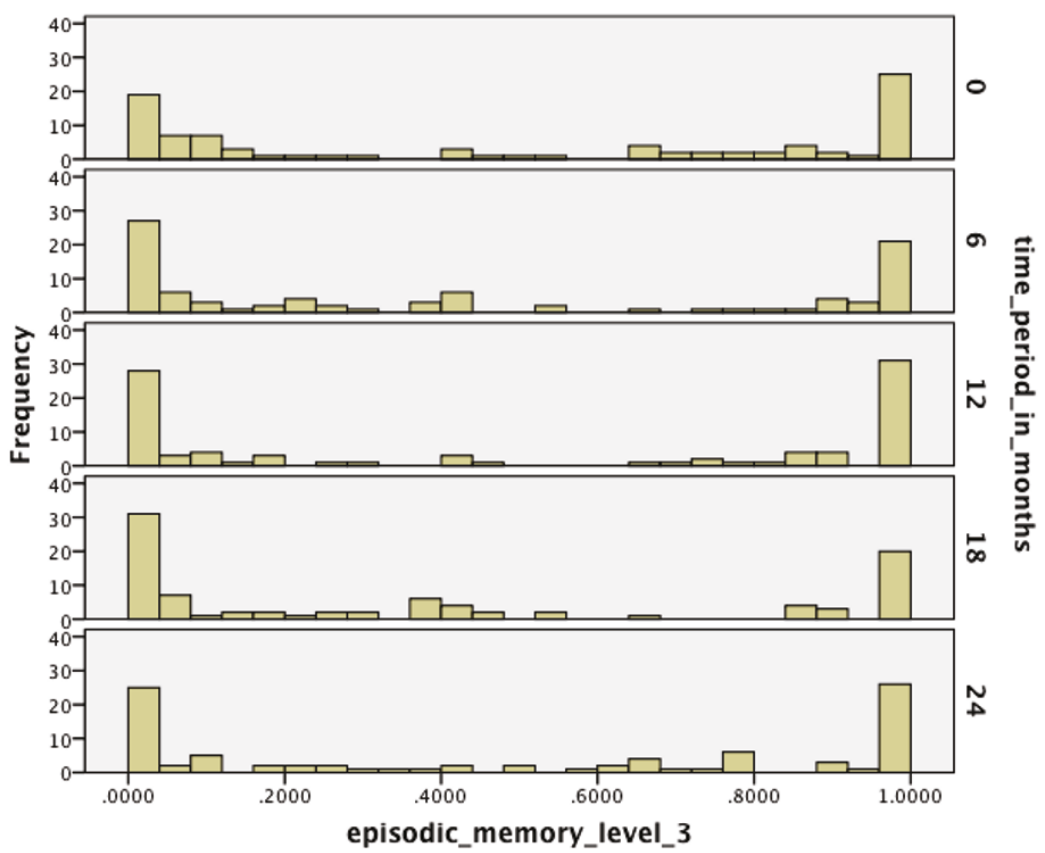

Figure 4 Probabilities of functioning over time among mild cognitive impairment (MCl) converters to Alzheimer's disease (AD) versus MCI non-converters. (a) Probability of relatively high functioning with episodic memory level 3 from 24 to 0 months before conversion to AD among $\mathrm{MCl}$ subjects. (b) Probability of relatively high functioning with episodic memory level 3 from baseline to 24 months among MCl subjects who did not convert within 36 months. 


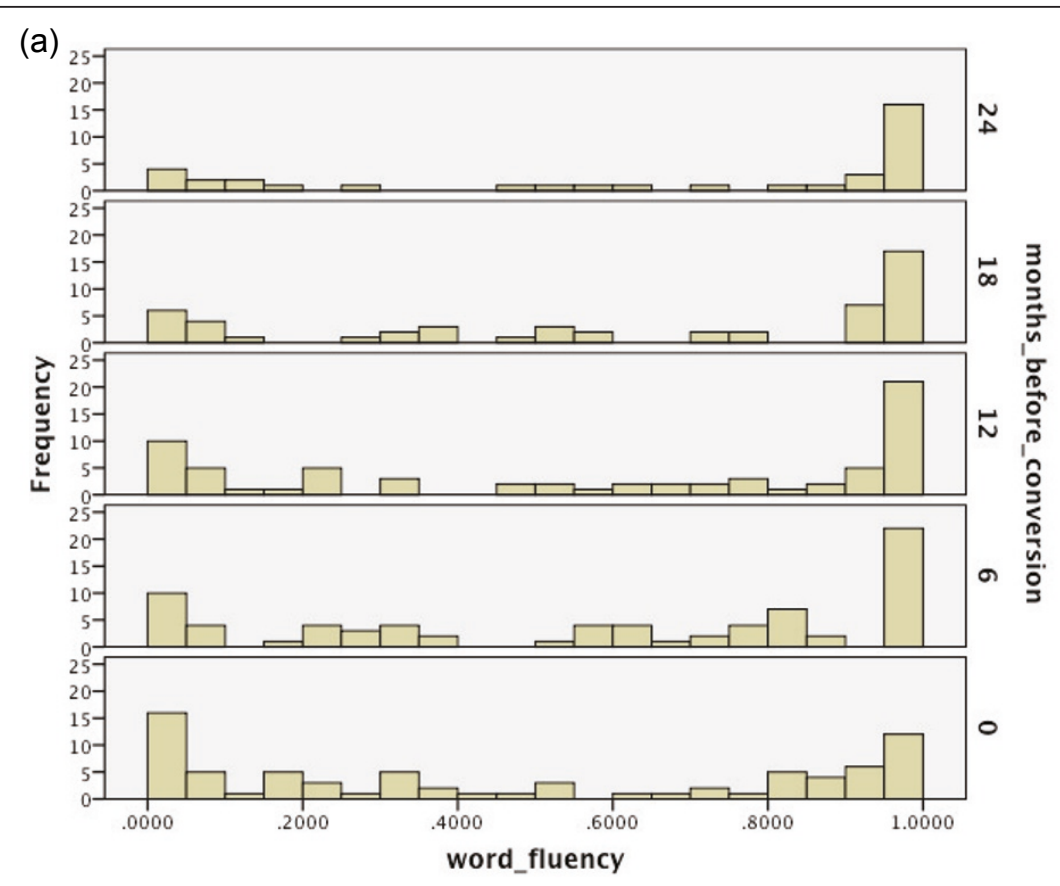

(b)

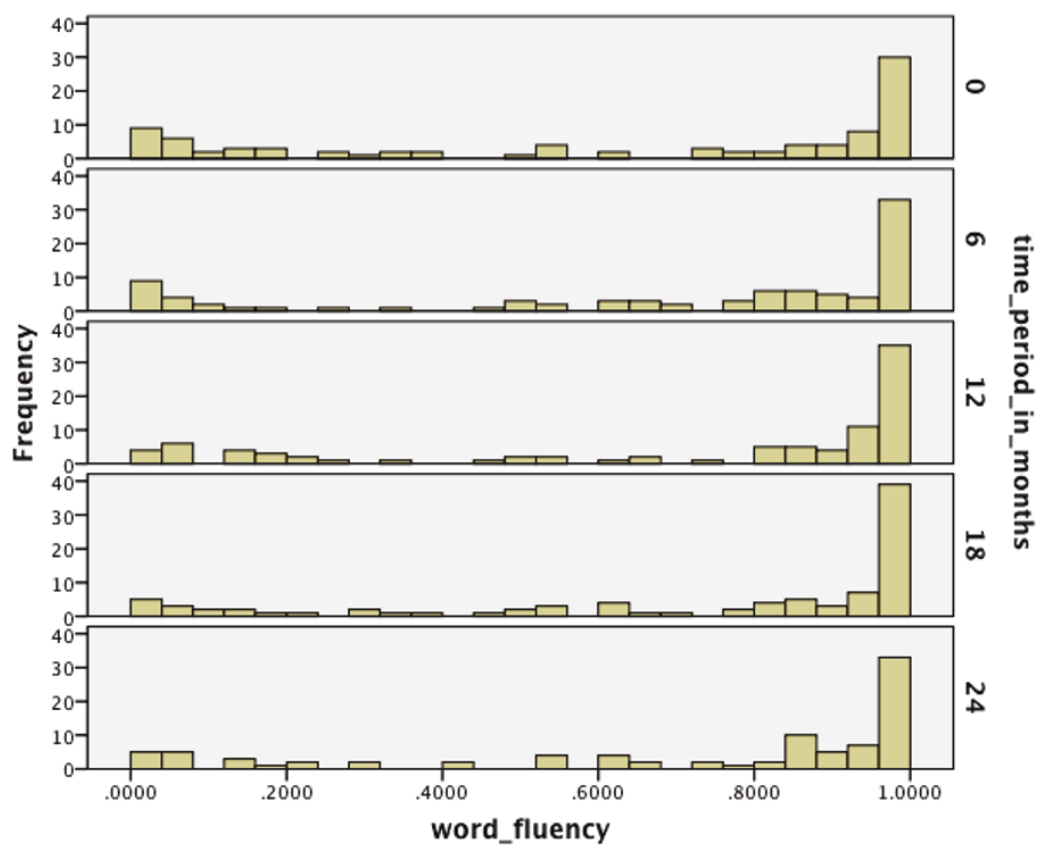

Figure 5 Probabilities of functioning over time among mild cognitive impairment (MCl) converters to Alzheimer's disease (AD) versus $\mathrm{MCl}$ non-converters. (a) Probability of relatively high functioning with word fluency from 24 to 0 months before conversion to $\mathrm{AD}$ among $\mathrm{MCl}$ subjects. (b) Probability of relatively high functioning with word fluency from baseline to 24 months among $\mathrm{MCl}$ subjects who did not convert within 36 months. 


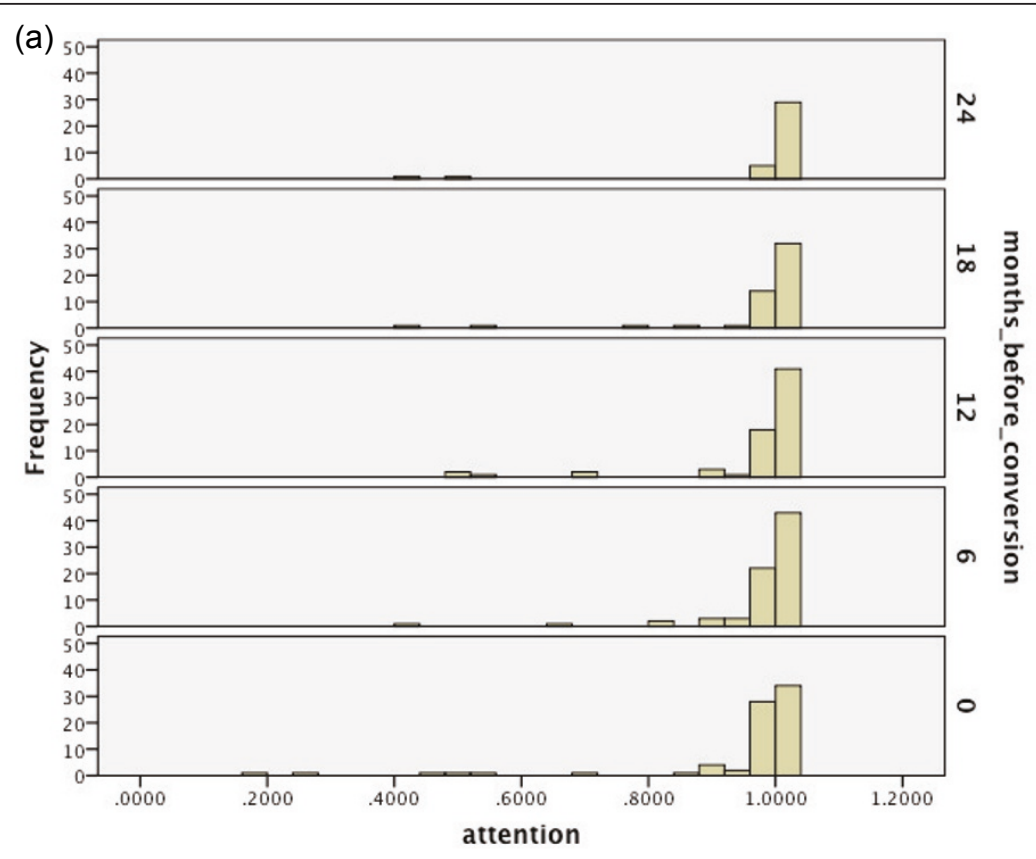

(b)

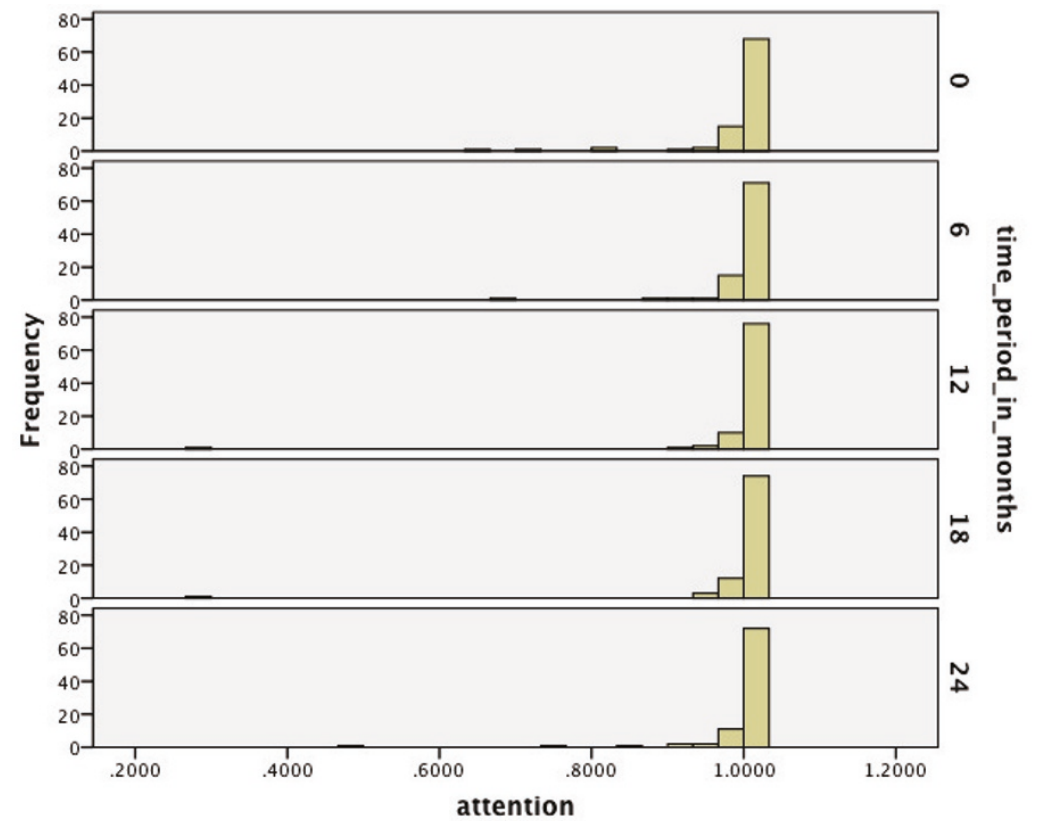

Figure 6 Probabilities of functioning over time among mild cognitive impairment (MCl) converters to Alzheimer's disease (AD) versus MCI non-converters. (a) Probability of relatively high functioning with attention/sustained vigilance from 24 to 0 months before conversion to $\mathrm{AD}$ among $\mathrm{MCl}$ subjects. (b) Probability of relatively high functioning with attention/sustained vigilance from baseline to $24 \mathrm{months}$ among $\mathrm{MCl}$ subjects who did not convert within 36 months. 


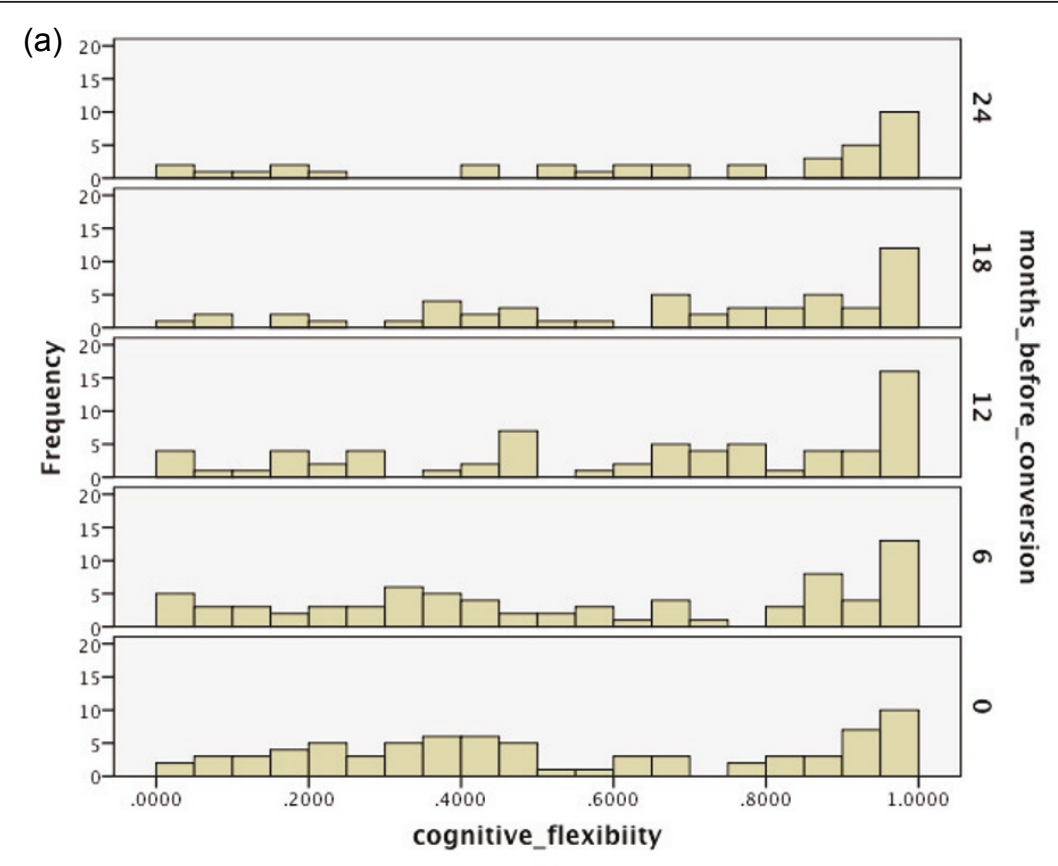

(b)

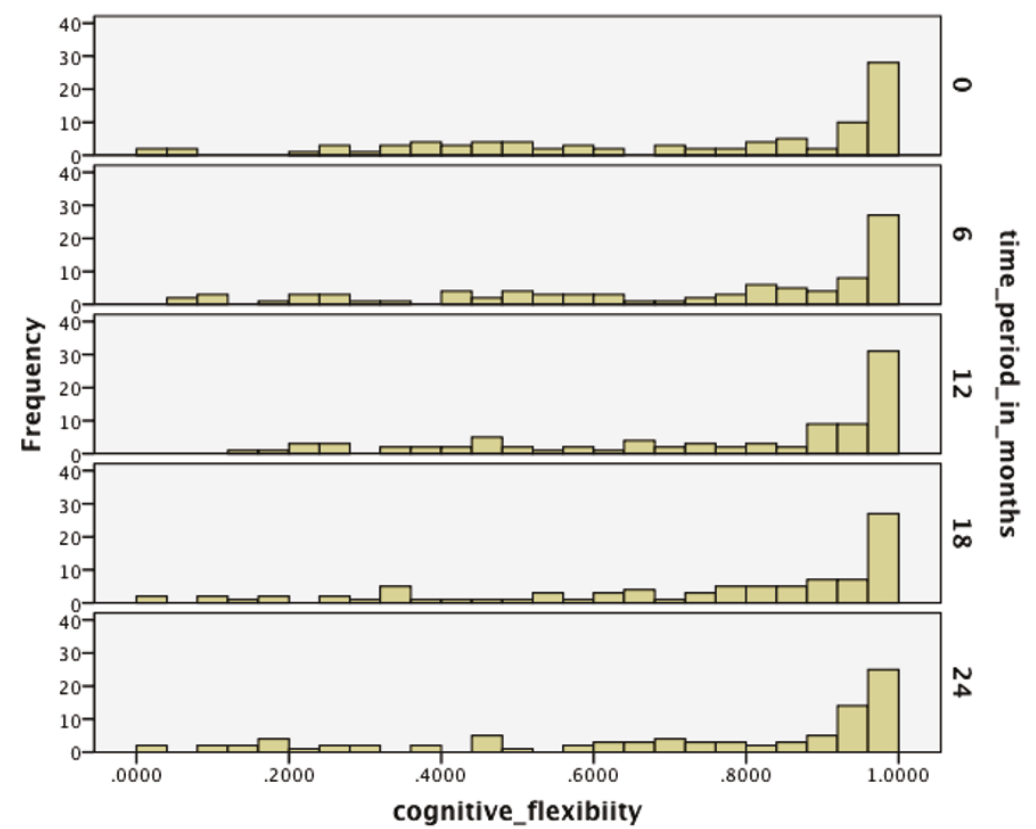

Figure 7 Probabilities of functioning over time among mild cognitive impairment (MCl) converters to Alzheimer's disease (AD) versus MCI non-converters. (a) Probability of relatively high functioning with cognitive flexibility from 24 to 0 months before conversion to AD

among $\mathrm{MCl}$ subjects. (b) Probability of relatively high functioning with cognitive flexibility from baseline to 24 months among $\mathrm{MCl}$ subjects who did not convert within 36 months. 


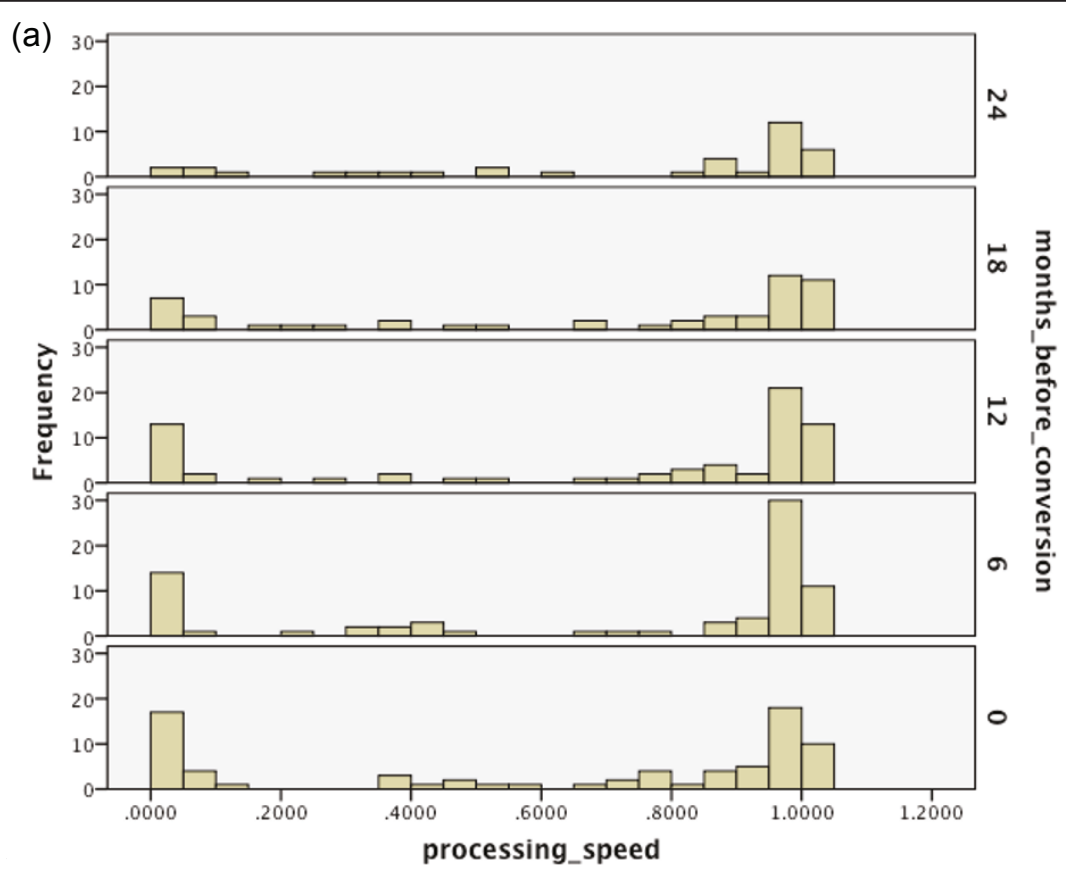

(b)

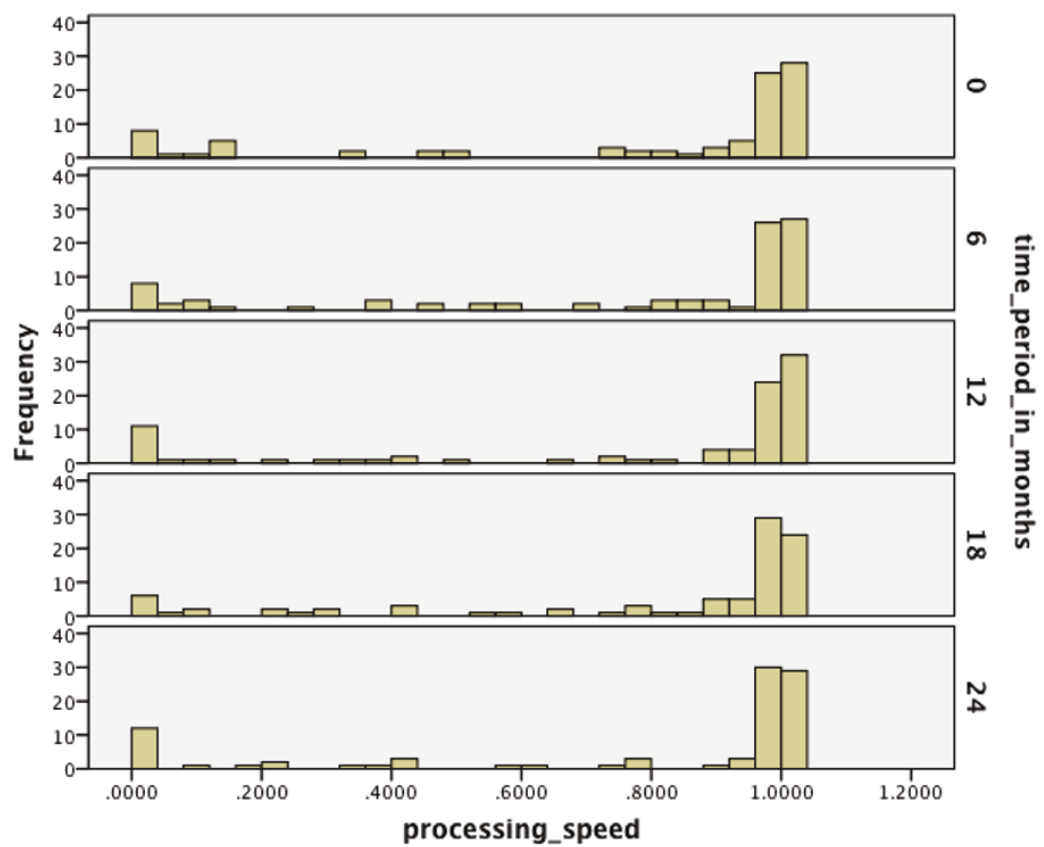

Figure 8 Probabilities of functioning over time among mild cognitive impairment (MCI) converters to Alzheimer's disease (AD) versus MCI non-converters. (a) Probability of relatively high functioning with perceptual motor speed from 24 to 0 months before conversion to AD among $\mathrm{MCl}$ subjects. (b) Probability of relatively high functioning with perceptual motor speed from baseline to 24 months among $\mathrm{MCl}$ subjects who did not convert within 36 months. 
In Figures $5 \mathrm{a}$ and $5 \mathrm{~b}$, it appears that there may be decline in word fluency values for a relatively small subset of subjects as they near conversion, but many converters also appear to retain high functioning. For non-converters, some subjects have lower functioning as well. Hence, word fluency does not appear useful for predicting conversion within a two-year period. In Figures $6 \mathrm{a}$ and $6 \mathrm{~b}$, note that there may be some slight decline in attention/ sustained vigilance for converters, but almost all subjects still retain high probability values for being at a high level of attention functioning.

In Figures $7 \mathrm{a}$ and $7 \mathrm{~b}$, there is definitely a fair amount of decline in values for cognitive flexibility among converters, although it is also not as pronounced as for episodic memory level 2. Also, some converters retain high functioning. Most non-converters retain high functioning over the duration. Hence, cognitive flexibility can be useful for discriminating future conversion outcomes, but does not appear as informative as episodic memory level 2. Finally, in Figures $8 \mathrm{a}$ and $8 \mathrm{~b}$, for perceptual motor speed, note that there appears to be a subset of converters for whom perceptual motor speed becomes more impaired. While there are non-converters who also have low probability values, this number is outweighed by the converters over the duration. Moreover, as Table 5 indicates, these converters are likely to also be relatively more impaired with episodic memory level 2 than the nonconverters. This allows us to identify this particular combination of lower level functioning as being specifically associated with high risk for conversion.

\section{Multivariate prediction using logistic regression models}

A multivariate model was fit that recognized the above findings, and included other variables such as gender, age, and educational level. The presence of an APOE4 allele was viewed as a binary variable, as well as whether or not a subject had attended college. Also, probability values for performing at a relatively high level for episodic memory level 2, cognitive flexibility, and perceptual motor speed were viewed as continuous explanatory variables. After an initial fit of a full model, gender, age, and educational level were clearly not significant predictors in the model (respective $P$-values were $0.96,0.65$, and 0.81; Wald's test). Using goodness-of-fit tests based on the test statistic of -2 times the difference in loglikelihood values to compare nested models, it appears the best fit is when episodic memory level 2, perceptual motor speed, and APOE4 status are included in the model. Results are given in Table 6 . Note that episodic memory level 2, perceptual motor speed, and APOE4 status all are significant predictors $(P$-value $<0.05$; Wald's test). When cognitive flexibility is included with these variables, it is not significant $(P$-value $=0.26)$.
Table 6 Multivariate logistic regression model with outcome as conversion to Alzheimer's disease (AD) from mild cognitive impairment $(\mathrm{MCl})$ within 24 months from baseline

\begin{tabular}{cccc}
\hline Explanatory variables & B & Wald test $P$-value & Exp (B) \\
\hline APOE4 & 0.623 & 0.024 & 0.536 \\
Episodic memory level 2 & -1.445 & 0.001 & 0.236 \\
Perceptual motor speed & -0.926 & 0.009 & 0.396 \\
Intercept & 1.193 & 0.000 & 3.298 \\
\hline
\end{tabular}

Dependent variable value of 1 indicates that a subject has converted to $A D$ from $\mathrm{MCl}$ within 24 months of baseline. Binary variable Apolipoprotein (APOE) $4=1$ indicates that the subject has at least one APOE4 allele and higher risk for conversion. Larger probability values of relatively high performance indicate lower risk for conversion.

Using the model-based estimated probabilities of converting to $\mathrm{AD}$ as a predictor, and for instance, using a cutoff value of 0.55 or higher, classification accuracy is $66.8 \%$, with positive predictive value of $61.5 \%$ (32 out of 52). Figure 9 displays receiver operating characteristic (ROC) curves for these logistic regression probabilities, as well as for the probabilities for episodic memory level 2 , perceptual motor speed, and cognitive flexibility. Values for the area under the curve (AUC) are 0.710, $0.678,0.655$, and 0.644 , respectively. Using a multivariate approach can thus apparently improve prediction. However, compared to relying upon episodic memory level 2 alone without the need for APOE4 status information, which would have practical appeal, the difference does not appear to be striking.

\section{Other prediction approaches}

A comprehensive review of research efforts using ADNI data is given in Weiner et al. (2012) [18]. This includes a description of work on prediction of MCI to AD conversion. Taking advantage of the richness of the ADNI data, prediction models have been developed based on a range of various imaging, cerebrospinal fluid, and genetic biomarkers, as well as cognition. Our prediction results appear to be comparable to non-cognitively oriented methods that rely on baseline data $[19,20]$. Advantages of a cognitive testing approach include noninvasiveness and cost, especially if focused and efficient NP batteries can be designed, and computer-based adaptive testing adopted in the future.

Other cognitive testing-based approaches to prediction include Tabert et al. (2006) [21] and Fleisher et al. (2007) [22]. Their prediction models depend directly on NP measurement scores, which in general may be difficult to interpret in terms of identifying which cognitive functions may be the source of poor scores. We believe that the results presented here add to these works, such as through a more specific consideration of multidomain MCI. Poset-based methods also provided insight into 


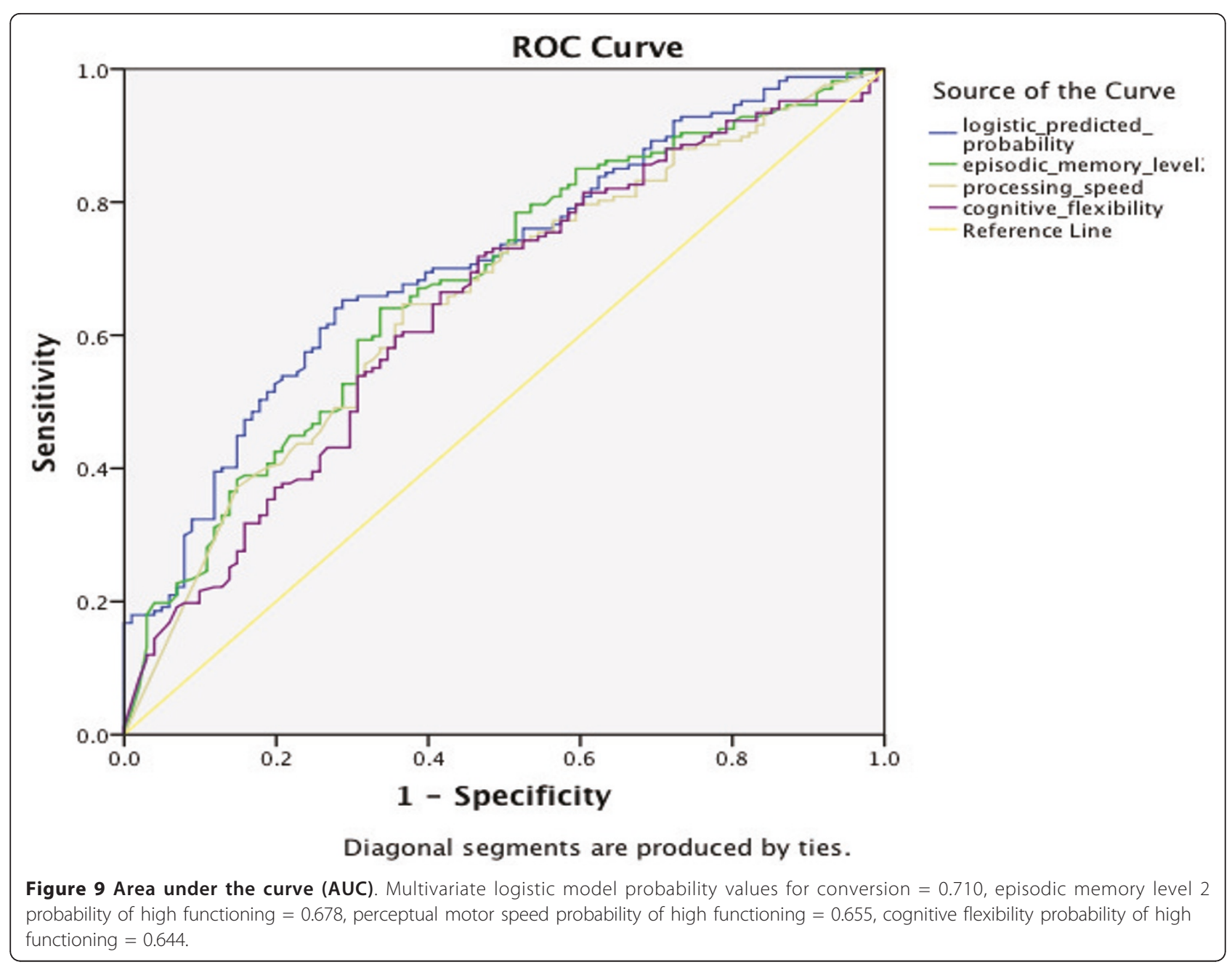

the course of cognitive change in MCI, by indicating how specific functions are affected over time. The findings depicted in Figure 4 allow for insight into the heterogeneity in cognitive progressions that arise among $\mathrm{MCI}$, and thus help in identifying profiles of high risk.

\section{Conclusions}

Our results suggest the utility of the poset-based approach in uncovering heterogeneity in risk for conversion from MCI to AD by generating subgroups tied to specific cognitive functions. Duration of 24 months from baseline measurement was considered. Among the cognitive functions evaluated, episodic memory was mostly strongly linked to conversion from $\mathrm{MCI}$ to $\mathrm{AD}$. This confirms similar findings in Tierney et al. (2005) [23], Tabert et al. (2006) [21], Blacker et al. (2007) [24], and Landau et al. (2010) [18]. We did find that cognitive flexibility and perceptual motor speed also is associated with conversion, as certain subjects are apparently affected in these domains during the 24 months preceding conversion. Conversely, MCI subjects with relatively less episodic memory impairment were observed to convert at a much lower rate. The importance of the APOE e4 allele in affecting risk for conversion is also clear $[18,19]$.

More precisely, it appears that in our model certain levels of episodic memory functioning are more discriminatory than others in terms of identifying MCI subjects at especially high risk for conversion. In particular, level-2 episodic memory functioning, more so than level-3, appears to best identify risk for conversion. It appears that delayed recall with distractors may be too difficult a task to discriminate risk very well. On the other hand, for those that can perform well at level 3 relative to others with $\mathrm{MCI}$, risk of conversion is much lower, as illustrated in Table 5.

Poset modeling also appears to be helpful in further clarifying the notion of multidomain MCI. Our analyses suggest that perceptual motor speed functioning may have a stronger link to subsequent risk of AD progression than other cognitive functions when considered in conjunction with relatively reduced function of level-2 
episodic memory. On the other hand, additional impairment with cognitive flexibility does not appear to increase risk beyond that due to episodic memory impairment. These results suggest that having relatively lower functioning across multiple functions can indeed increase risk for $\mathrm{AD}$, but, that it may matter which of the functions are impaired. Because corresponding samples become smaller as specific combinations of deficits are analyzed, however, these analyses must be viewed as preliminary.

A possible concern is that the ranges of performance levels for functions in the poset model are limited, particularly when a functioning level is either high or low. However, it should be kept in mind that there are also limited NP response data available, due to the time-consuming and burdensome nature of NP measurement. Hence, there are statistical limitations to the granularity of information on functioning that can be assessed accurately. Our model was shown to be feasible, while still being able to provide discriminating information relating to AD conversion. Another limitation is the scope of inference that can be made from the ADNI sample, which was overwhelmingly Caucasian, and imbalanced towards males. Certainly, these findings should be validated in other datasets.

\section{Future directions}

Since the poset model allows for precise and detailed cognitive profiles, this approach can be used in conjunction with imaging and genetic studies. As an example, a cluster analysis approach has recently been applied to characterize MCI subgroups by cognitive characteristics [25]. While white matter lesion burden was found to differ by these groupings, in general, cognitive subtypes resulting from cluster analysis can at times be more difficult to interpret than profiles generated with poset models. Also, note the persistent heterogeneity of conversion outcomes among MCI subjects even within the cognitively homogeneous subgroups generated here. These subgroups could be an interesting basis for functional magnetic resonance imaging (fMRI) studies of cognitive reserve, which may improve understanding of how this heterogeneity arises [26,27]. Poset modeling may also be useful in clinical trial design, as enriched sampling from subgroups of MCI subjects that have high positive predictive value of conversion could reduce sample size requirements.

Finally, poset models can serve as a basis for adaptive NP testing, with NP measures being selected for administration dynamically, based on the responses that have already been observed $[7,8]$. As demonstrated, an attractive feature of poset models is that since they are comprised of discrete states, accurate statistical classification can be conducted with relatively few measures. Adaptive tests can further reduce subject burden and allow for more focused and efficient testing, which in turn would enhance the appeal of cognitive testing for prediction.

\section{Additional material}

Additional file 1: Appendix: Statistical framework for data analysis and model validation. This file contains statistical details relating to the poset modeling, including parameter estimates and classification summaries. It also describes how model validation was conducted.

\section{Abbreviations}

AD: Alzheimer's disease; ADAS-Cog: Alzheimer's disease assessment scalecognitive; ADNI: Alzheimer's Disease Neuroimaging Initiative; APOE: Apolipoprotein E; AUC: area under the curve; AVLT: auditory verbal learning test; fMRI, functional magnetic resonance imaging; $\mathrm{MCl}$ : mild cognitive impairment; MMSE: mini-mental status exam; NP: neuropsychological; poset: partially ordered set; ROC; receiver operator characteristic; WAIS-R: Wechsler adult intelligence scale-revised.

\section{Authors' contributions}

$C T$ developed the statistical methods, conducted statistical analyses, and helped prepare the manuscript; HYT conducted analyses; JJ helped frame and conduct the analyses; FV programmed the software, helped in developing the statistical methods, and helped in the analysis; MS helped in the analyses; TY conducted analyses; KS helped in the analyses and prepared the manuscript; AL helped frame and conduct the analyses, and prepare the manuscript. All authors have read and approved the manuscript for publication. Data used in the preparation of this article were obtained from the ADNI database [28]. As such, the investigators within the ADNI contributed to the design and implementation of ADNI and/or provided data but did not participate in analysis or writing of this report. ADNI investigators include a complete listing available at [29].

\section{Competing interests}

Dr Tatsuoka was funded in part by AstraZeneca Pharmaceuticals to do this research, and has written a related patent. Drs Tseng, Varadi, Smyth and Yamada have no conflicts of interest to report. Dr Jaeger was Director, Global Medicines Development, Neuroscience, AstraZeneca Pharmaceuticals during the funding of this research, and is now with CogState, Inc. Dr Smith was a consultant for Anavex Life Sciences Corporation, Medivation, Eisai, Glaxo Welcome Kline, and Neurotez; he owns stock options in Neurotez, Aria, Panancea, and Curaxis Pharmaceuticals. Dr Lerner receives research support from Forest Labs, Pfizer, Medivation, Baxter Labs, and Avid Pharmaceuticals.

\section{Acknowledgements}

This work was funded in part by the grants from AstraZeneca Pharmaceuticals and The G.R. Lincoln Family Foundation. Data collection and sharing for this project was funded by the ADNI (National Institutes of Health Grant U01 AG024904). ADNI is funded by the National Institute on Aging, the National Institute of Biomedical Imaging and Bioengineering, and through generous contributions from the following: Abbott, AstraZeneca $A B$, Bayer Schering Pharma AG, Bristol-Myers Squibb, Eisai Global Clinical Development, Elan Corporation, Genentech, GE Healthcare, GlaxoSmithKline, Innogenetics, Johnson and Johnson, Eli Lilly and Co., Medpace, Inc., Merck and Co., Inc., Novartis AG, Pfizer Inc, F. Hoffman-La Roche, Schering-Plough, Synarc, Inc., as well as non-profit partners, the Alzheimer's Association and Alzheimer's Drug Discovery Foundation, with participation from the US Food and Drug Administration. Private sector contributions to ADNI are facilitated by the Foundation for the National Institutes of Health [30]. The grantee organization is the Northern California Institute for Research and Education, and the study is coordinated by the Alzheimer's Disease Cooperative Study at the University of California, San Diego. ADNI data are disseminated by the Laboratory for Neuroimaging at the University of California, Los Angeles. This research was also supported by NIH grants P30 AG010129, K01 AG030514, and the Dana Foundation. 
Alzheimer's Disease Neuroimaging Initiative (ADNI): 'Data used in the preparation of this article were obtained from the Alzheimer's Disease Neuroimaging Initiative (ADNI) database [28]. The ADNI was launched in 2003 by the National Institute on Aging (NIA), the National Institute of Biomedical Imaging and Bioengineering (NIBIB), the Food and Drug Administration (FDA), private pharmaceutical companies and non-profit organizations, as a $\$ 60$ million, 5-year public private partnership. The primary goal of ADNI has been to test whether serial magnetic resonance imaging (MRI), positron emission tomography (PET), other biological markers, and the progression of mild cognitive impairment (MCl) and early Alzheimer's disease (AD). Determination of sensitive and specific markers of very early $A D$ progression is intended to aid researchers and clinicians to develop new treatments and monitor their effectiveness, as well as lessen the time and cost of clinical trials. The Principal Investigator of this initiative is Michael W Weiner, MD, VA Medical Center and University of California - San Francisco. $\mathrm{ADNl}$ is the result of efforts of many co-investigators from a broad range of academic institutions and private corporations, and subjects have been recruited from over 50 sites across the US and Canada. The initial goal of ADNI was to recruit 800 adults, ages 55 to 90 , to participate in the research approximately 200 cognitively normal older individuals to be followed for 3 years, 400 people with $\mathrm{MCl}$ to be followed for 3 years and 200 people with early $A D$ to be followed for 2 years.' For up-to-date information, see [31].

\section{Author details}

${ }^{1}$ Department of Neurology, Case Western Reserve University, 11100 Euclid Avenue, Cleveland, $\mathrm{OH} 44106$ USA. ${ }^{2}$ Neurological Institute, University Hospitals Case Medical Center, 3619 Park East Drive, Beachwood, OH 44122 USA. ${ }^{3}$ Department of Epidemiology and Biostatistics, Case Western Reserve University, 10900 Euclid Avenue, Cleveland, OH 44106 USA. ${ }^{4}$ Department of Human Development, Teachers College, Columbia University, 525 West $120^{\text {th }}$ Street, New York, NY 10027 USA. ${ }^{5}$ AstraZeneca Pharmaceuticals, Clinical Development, Neuroscience, 1800 Concord Pike, Wilmington, DE 19807 USA. ${ }^{6}$ Department of Psychiatry and Behavioral Sciences, Albert Einstein College of Medicine, 1300 Morris Park Avenue, Bronx, NY 10461 USA. ${ }^{7}$ Tanar Software, Hunting Valley, OH 44022 USA. ${ }^{8}$ Department of Pathology, Case Western Reserve University, 2103 Cornell Road, Cleveland, OH 44106 USA.

\section{Received: 30 May 2012 Revised: 1 February 2013}

Accepted: 6 February 2013 Published: 6 March 2013

\section{References}

1. Petersen RC, Doody R, Kurz A, Mohs RC, Morris JC, Rabins PV, Ritchie K, Rossor M, Thal L, Winblad B: Current concepts in mild cognitive impairment. Arch Neurol 2001, 58:1985-1992.

2. Petersen RC, Thomas RG, Grundman M, Bennett D, Doody R, Ferris S, Galasko D, Jin S, Kaye J, Levey A, Pfeiffer E, Sano M, van Dyck CH, Thal L, Alzheimer's Disease Cooperative Study Group: Vitamin E and donepezil for the treatment of mild cognitive impairment. N Engl J Med 2005, 352:2379-2388.

3. Andrieu S, Coley N, Aisen P, Carrillo MC, DeKosky S, Durga J, Fillit H, Frisoni GB, Froelich L, Gauthier S, Jones R, Jönsson L, Khachaturian Z, Morris JC, Orgogozo JM, Ousset PJ, Robert P, Salmon E, Sampaio C, Verhey $F$, Wilcock $G$, Vellas $B$ : Methodological issues in primary prevention trials for neurodegenerative dementia. J Alzheimers Dis 2009, 16:235-270.

4. Petersen RC: Mild cognitive impairment as a diagnostic entity. J Intern Med 2004, 256:183-194.

5. Rosen WG, Mohs RC, Davis KL: A new rating scale for Alzheimer's disease. Am J Psychiatry 1984, 141:1356-1364.

6. Folstein MF, Folstein SE, McHugh PR: "Mini-mental state". A practical method for grading the cognitive state of patients for the clinician. J Psychiatr Res 1975, 12:189-198.

7. Tatsuoka C, Ferguson T: Sequential classification on partially ordered sets. Journal of the Royal Statistical Society, Series B 2003, 65:143-157.

8. Tatsuoka C: Data analytic methods for latent partially ordered classification models. Journal of the Royal Statistical Society, Series C (Applied Statistics) 2002, 51:337-350, See also Corrigendum 2005, 54:465-467.

9. Aisen PS, Petersen RC, Donohue MC, Gamst A, Raman R, Thomas RG, Walter S, Trojanowski JQ, Shaw LM, Beckett LA, Jack CR Jr, Jagust W, Toga AW, Saykin AJ, Morris JC, Green RC, Weiner MW, Alzheimer's Disease Neuroimaging Initiative: Clinical core of the Alzheimer disease neuroimaging initiative: Progress and plans. Alzheimers Dement 2010, 6:239-246.

10. Jaeger J, Tatsuoka C, Berns S, Varadi F, Czobor P, Uzelac S: Associating functional recovery with neurocognitive profiles identified using partially ordered classification models. Schizophr Res 2006, 85:40-48.

11. Jaeger J, Tatsuoka C, Berns SM, Varadi F: Distinguishing neurocognitive functions in schizophrenia using partially ordered classification models. Schizophr Bull 2006, 32:679-691.

12. Landau SM, Harvey D, Madison CM, Reiman EM, Foster NL, Aisen PS, Petersen RC, Shaw LM, Trojanowski JQ, Jack CR Jr, Weiner MW, Jagust WJ, Alzheimer's Disease Neuroimaging Initiative: Comparing predictors of conversion and decline in mild cognitive impairment. Neurology 2010, 75:230-238.

13. Aggarwal NT, Wilson RS, Beck TL, Bienias JL, Berry-Kravis E, Bennett DA: The apolipoprotein E epsilon4 allele and incident Alzheimer disease in persons with mild cognitive impairment. Neurocase 2005, 11:3-7.

14. Tatsuoka C: Sequential classification on partially ordered sets. PhD thesis Cornell University, Statistics Department; 1996.

15. Ishwaran H, James LF: Approximate Dirichlet process computing in finite normal mixtures: Smoothing and prior information. J Comp Graph 2002, 97:1154-1166

16. Tatsuoka C, Varadi F, Jaeger J: Latent partially ordered classification models and normal mixtures. Journal of Educational and Behavioral Statistics.

17. Geweke J: Evaluating the accuracy of sampling-based approaches to the calculation of posterior moments. In Bayesian Statistics 4. Edited by: Bernardo JM, Berger J, Dawid AP, Smith AFM. Oxford, UK: Oxford University Press; 1992:

18. Weiner MW, Veitch DP, Aisen PS, Beckett LA, Cairns NJ, Green RC, Harvey D, Jack CR, Jagust W, Liu E, Morris JC, Petersen RC, Saykin AJ, Schmidt ME, Shaw L, Siuciak JA, Soares H, Toga AW, Trojanowski JQ, Alzheimer's Disease Neuroimaging Initiative: The Alzheimer's Disease Neuroimaging Initiative: A review of papers published since its inception. Alzheimer's \& Dementia 2012, 8(Suppl):S1-S68.

19. Querbes O, Aubry F, Pariente J, Lotterie JA, Demonet JF, Duret V, Puel M, Berry I, Fort JC, Celsis P, Alzheimer's Disease Neuroimaging Initiative: Early diagnosis of Alzheimer's disease using cortical thickness: impact of cognitive reserve. Brain 2009, 132:2036-2047.

20. Ewers M, Walsh C, Trojanowski JQ, Shaw LM, Petersen RC, Jack CR Jr, Feldman $\mathrm{HH}$, Bokde AL, Alexander GE, Scheltens P, Vellas B, Dubois B, Weiner M, Hampel H, North American Alzheimer's Disease Neuroimaging Initiative (ADNI): Prediction of conversion from mild cognitive impairment to Alzheimer's disease dementia based upon biomarkers and neuropsychological test performance. Neurobiol Aging 2012, 33:1203-1214.

21. Tabert MH, Manly JJ, Liu X, Pelton GH, Rosenblum S, Jacobs M, Zamora D, Goodkind M, Bell K, Stern Y, Devanand DP: Neuropsychological prediction of conversion to Alzheimer disease in patients with mild cognitive impairment. Arch Gen Psychiatry 2006, 63:916-24.

22. Fleisher AS, Sowell BB, Taylor C, Gamst AC, Petersen RC, Thal LJ: Clinical predictors of progression to Alzheimer disease in amnestic mild cognitive impairment. Neurology 2007, 68:1588-1595.

23. Tierney MC, Yao C, Kiss A, McDowell I: Neuropsychological tests accurately predict incident Alzheimer disease after 5 and 10 years. Neurology 2005, 64:1853-1859.

24. Blacker D, Lee H, Muzikansky A, Martin EC, Tanzi R, McArdle JJ, Moss M, Albert M: Neuropsychological measures in normal individuals that predict subsequent cognitive decline. Arch Neurol 2007, 64:862-871.

25. Delano-Wood L, Bondi MW, Sacco J, Abeles N, Jak AJ, Libon DJ, Bozoki A: Heterogeneity in mild cognitive impairment: Differences in neuropsychological profile and associated white matter lesion pathology. J Int Neuropsychol Soc 2009, 15:906-914.

26. Stern Y: Cognitive reserve and Alzheimer disease. Alzheimer Dis Assoc Disord 2006, 20(Suppl 2):S69-S74.

27. Stern Y: Cognitive reserve. Neuropsychologia 2009, 47:2015-2028.

28. Alzheimer's Disease Neuroimaging Initiative (ADNI) database. [http:// www.loni.ucla.edu/ADNI].

29. Alzheimer's Disease Neuroimaging Initiative (ADNI) investigators. [http:// www.loni.ucla.edu/ADNI/Collaboration/ADNI_Authorship_list.pdf].

30. Foundation for the National Institutes of Health. [http://www.fnih.org]. 
31. Alzheimer's Disease Neuroimaging Initiative (ADNI). [http://www.adniinfo.org].

doi:10.1186/alzrt168

Cite this article as: Tatsuoka et al:: Modeling the heterogeneity in risk of progression to Alzheimer's disease across cognitive profiles in mild cognitive impairment. Alzheimer's Research \& Therapy 2013 5:14.

Submit your next manuscript to BioMed Central and take full advantage of:

- Convenient online submission

- Thorough peer review

- No space constraints or color figure charges

- Immediate publication on acceptance

- Inclusion in PubMed, CAS, Scopus and Google Scholar

- Research which is freely available for redistribution

Submit your manuscript at www.biomedcentral.com/submit
() Biomed Central 